\title{
Distribution of the McKenzie Syndromes and Pelvic Girdle Pain in Pregnancy-related Back Pain
}

\author{
Ayanniyi O., Sanya A.O. \\ Department of Physiotherapy, College of Medicine, University of Ibadan/ University College Hospital (UCH), \\ Ibadan, Nigeria \\ Correspondence \\ Dr. Olusola Ayanniyi, Physiotherapy Department, College of Medicine, University of Ibadan/ UCH, Ibadan, \\ Nigeria・Email: drayanniyi@gmail.com
}

\begin{abstract}
SUMMARY
This study investigated the distribution of the three McKenzie syndromes and pelvic girdle pain (PGP) among participants with pregnancy-related back pain (BP) attending some selected medical facilities. The aim was to determine if pregnancy-related BP can be classified into the three McKenzie syndromes and also to find out the distribution pattern of PGP among the participants.

Participants were recruited into the study consecutively and were assessed using the McKenzie assessment protocol and pelvic girdle pain provocative tests. Participants with low back pain (LBP) or high back pain (HBP) were classified into the three McKenzie syndromes and those with PGP were classified according to their pain site. Data were analysed using descriptive and inferential statistics. Alpha level was set at 0.05 .

The participants with HBP had the lowest mean age $(26.2 \pm 5.6$ years $)$. There was a significant difference in the mean age of the participants across the three $B P$ groups $(F=3.9525 ; p=0.0199)$. Among the participants with LBP, derangement syndrome constituted $95.7 \%$ and postural syndrome $4.3 \%$. All the participants with HBP had derangement syndrome. Bilateral pain was present in $62 \%$ of the participants with PGP.

Derangement syndrome was predominant among participants with LBP and HBP while bilateral pain presentation is prevalent among the participants with PGP.

It is recommended that the McKenzie approach should be promoted and used in the physical assessment of women with pregnancy-related back pain.
\end{abstract}

Keywords: McKenzie syndromes, pelvic girdle pain, pregnancy-related back pain

\section{INTRODUCTION}

McKenzie syndrome is a clinical terminology that was developed to describe distinct patterns of symptom presentation and classification among people suffering from back pain (BP) of mechanical origin (McKenzie and May, 2003). According to the McKenzie school of thought, three syndromes are described, namely postural, dysfunction and derangement syndromes. Each of the syndromes is totally different from the others, therefore, each syndrome is treated as a separate entity (McKenzie and May, 2003). Postural syndrome is characterized by intermittent pain brought about only by prolonged static loading of normal tissues while dysfunction syndrome is characterized by intermittent BP which appears only at the end range of movement when the affected spinal structures are mechanically loaded. Derangement syndrome is defined as a painful condition brought about by anatomical disruption or displacement of tissues within the intervertebral disc. Pain from derangement syndrome is often constant and exhibits itself during repeated movement (McKenzie and May, 2003).

$\mathrm{BP}$ is recognized as one of the most prevalent musculoskeletal problems associated with pregnancy (Mogren and Pohjanen, 2005). This type of BP is referred 
to as pregnancy-related because it has its onset in pregnancy. It often appears around 22 weeks of gestation, but it may also occur at any time during pregnancy ((Mogren and Pohjanen, 2005). Back pain refers to symptoms of pain/discomfort perceived to originate from structures of the pelvic joints, and the lumbar and thoracic spine regions of the human back (Hipp et al, 1989; McKenzie and May, 2003). Low back pain (LBP) refers specifically to symptoms of pain/discomfort originating from the lumbar spine region with or without radiation of pain to the gluteal fold and exhibiting a negative response to pelvic girdle pain (PGP) provocation tests (Hipp et al, 1989; Ostgaard et al, 1994). High back pain (HBP) refers specifically to symptoms of pain/discomfort originating from the thoracic spine region, with or without radiation of pain to the chest wall (Cyriax, 1978; McKenzie, 1990). McKenzie (1980) stated that both during and after pregnancy, women are subjected to alter mechanical stresses, which affect the back and often result in a back problem. Pelvic girdle pain (PGP) on the other hand is peculiar to the female gender. PGP is described as deep, stabbing, unilateral or bilateral, recurrent or continuous pain presenting between the posterior iliac crest and the gluteal fold, it was previously called posterior pelvic pain (Ostgaard et al, 1994; Wu et al, 2004; Vermani et al, 2010). PGP is common during pregnancy and the postpartum period (Ostgaard et al, 1994, Vleeming et al, 2008).

To diagnose PGP, Albert et al (2000) advocated using a combination of provocative pelvic joint tests whereas a multitude of clinical tests is recommended for the diagnosis of pregnancy-related LBP and HBP due to the uncertainty of their nature. However, it is opined that pregnancy-related LBP and HBP have common features with the mechanical LBP and HBP seen in the general population (Ostgaard et al, 1996; Sturesson et al, 1997; Lile et al, 2003). In the light of the above observation, this study was designed to find out if pregnancy-related LBP and HBP can be classified into the three McKenzie syndromes for the purpose of assessment, management and to determine the pattern of presentation of PGP among the participants. Data for the study was derived from a larger study conducted on the prevalence of BP among pregnant women and available management approaches during pregnancy in some selected medical facilities in Ogbomoso and Ibadan, Oyo State, Nigeria.

\section{MATERIALS AND METHODS}

\section{Participants and study time}

Pregnant women were recruited from five selected antenatal centres in Ogbomoso and Ibadan, Oyo State, Nigeria. The study was conducted over a period of two years and three months.

\section{Instruments}

The following instruments were used:

a. Plinth: A firm strong plinth was used for examination of the participants.

b. Chair: A firm strong and straight-backed chair was used for assessment in sitting.

\section{Procedure}

Ethical approval for the study was granted by the Ethics Committees of the medical facilities where the study was carried out. Permission was also obtained from the various heads of the antenatal clinics where the study was conducted. The research assistants recruited for the study attended two training sessions of one hour each to make them familiar with their expected role during the study.

\section{Participants and Setting}

Pregnant women with back pain problem were purposely recruited into the study from the antenatal units of five medical centres. The participants took part in the study with the full consent of their primary antenatal care providers who were responsible for medical screening and provision of information on the clinical status of their patients. The rationale and procedure for the study were explained to the participants and their informed consent to take part in the study was sought and obtained. The right of any participants to decline participation in the study was explained to them before commencing the study. Participants were recruited into the study as they became available during the course of the study. Screening space was purposely secured in the participating centres to attend to the participants.

\section{Inclusion criteria}

The inclusion criteria for this study are as follows:

(I) Pregnant women who were registered in the antenatal clinics of the selected medical facilities where the study was conducted and who were willing to take part in the study. 
(ii) A good state of health with no earlier history of compromised pregnancy and no problem in the current pregnancy.

(iii) Women who were able to understand English or Yoruba language which were the mediums of communication.

\section{Exclusion criteria}

The exclusion criteria for this study are as follows:

(I) Pregnant women who were unable to understand English or Yoruba language, which were the mediums of communication, were excluded from the study.

(ii) Participants' with red flags, indicative of serious spinal pathology with signs and symptoms of nerve root compression with at least two signs present, such as dermatomal sensory loss, myotonic muscle weakness and reduced lower limb reflexes, were excluded from the study. Also excluded from this study were participants with LBP/HBP whose symptoms behaviour did not fit into the pattern described by McKenzie and May (2003).

(iii) Participants that were not recommended by their primary antenatal care providers due to their perceived health status.

\section{Screening of the participants}

Participants were assessed individually by a physical therapist trained and certified in the McKenzie method. The assessment covered all aspects of physical examination and each participant's back pain status was checked by medical history, interview, and clinical/physical examination. Participants with pain in the lumbar or thoracic spine region were assessed using the McKenzie assessment format. The assessment protocol has being found to be reliable and valid when carried out by trained practitioners (Razmjou et al, 2000; Fritz et al, 2000) ${ }^{\circledR}=0.70-1.00$ ), (validity 0.92 ). The repeated movement testing part of the McKenzie method of assessment was modified to include a series of fewer repetitions per bout (two to three repetitions) instead of the usual series of 10 repetitions per bout. A resting period of 30 seconds was observed between each bout of repeated movement testing. The repeated movement testing was carried out as adjudged necessary by the examiner without inducing fatigue in the participants. The repeated movement testing was used to assess the participant's underlying mechanical syndrome. Fewer repetitions per bout were opted for in order to ameliorate musculoskeletal laxity associated with pregnancy (MacFayden, 1989). This was carried out for flexion in standing/sitting and extension in standing for LBP. For the assessment of participants with HBP, flexion in high sitting and extension in high sitting procedures were used while sustained positioning was used to assess for the postural syndrome. The participants' directional preferences in response to McKenzie evaluative procedures were also noted and recorded. According to McKenzie and May (2003) directional preference is defined as: "a single direction of posture or movement that decreases, centralizes, or abolishes symptoms and typically eliminates prior limitation of movement".

Participants with suspected pelvic girdle pain (PGP) were also subjected to the McKenzie assessment procedure and their LBP type was later confirmed using a combination of four provocative tests (Posterior shear/Thigh thrust test, Faber/Patrick's test, Distraction or the Gapping test, Compression test) as described by Albert et al (2000). Positive findings on at least three of the four tests confirmed the pelvic girdle structures as a probable source of the participant's back pain.

\section{Classification of the participants}

Based on the overall clinical picture of the participants' history and symptoms behaviour during and after the test movements, participants with HBP and LBP were categorized into one of the three McKenzie syndromes namely, postural, dysfunction and derangement. The participants with derangement syndrome were further assessed for directional preference. Participants with suspected PGP presentation with at least three positive provocative tests were categorized into unilateral or bilateral PGP according to the site of their pain.

\section{Data analysis}

Data generated from each of the medical facilities were pooled for analysis. The following data analyses were carried out: descriptive statistics of percentage, mean and standard deviation were computed for age, type of syndrome identified and site of pelvic girdle pain. One-way analysis of variance (ANOVA) was carried out to study the pattern of distribution of mean age among the participants. The level of significance was set at $0.05 \alpha$

\section{RESULTS}

A total of 466 participants who satisfied the inclusion criteria were classified into the three BP groups: LBP 
(230), HBP (86), and PGP (150). Table 1 shows the pattern of distribution of mean age of the participants in the three groups. Participants with HBP had the lowest mean age $(26.2 \pm 5.2)$. One-way analysis of variance for mean age among the participants in the three groups revealed a significant difference across the groups $(\mathrm{F}=3.9525 ; \mathrm{p}=$ 0.0199). Turkey HSD post-hoc test shows that a significant difference exists between the mean age of the HBP group and the mean age of the PGP group $(p=0.0220)$.

Table 1. One-way analysis of variance comparison of the mean age of the participants in the three back pain groups $(\mathrm{N}=466)$

\begin{tabular}{lll}
\hline Back Pain Group & $\mathrm{n}(\%)$ & Mean Age \pm SD \\
\hline LBP & $230(49.4 \%)$ & $27.0 \pm 5.6$ \\
HBP & $86(18.4 \%)$ & $26.2 \pm 5.2$ \\
PGP & $15032.2 \%)$ & $28.2 \pm 5.7$ \\
\hline F-ratio & & 3.9525 \\
p-level & $0.0199 *$ \\
$\begin{array}{l}\alpha=0.05 \\
\text { Key: LBP = Low Back Pain; } \\
\text { PGP = Pelvic Girdle Pain; }\end{array}$ & $\begin{array}{l}\text { HBP }=\text { High Back Pain; } \\
\%=\text { Percentage; }\end{array}$ & SD $=$ Standard deviation \\
$*=$ Significant &
\end{tabular}

The distribution of the McKenzie syndromes among the participants with LBP and HBP, together with the pattern of pain site distribution among the participants with PGP are as shown in table 2 . No case of dysfunction syndrome was identified among the participants with LBP while all the participants with HBP had only derangement syndrome and showed a directional preference for extension only. However, 199(86.5\%) participants with LBP showed a directional preference for extension while $31(13.5 \%)$ participants showed a directional preference for flexion. Of the 150 participants with PGP, 41(27.3\%) showed a directional preference for flexion and $37(24.7 \%)$ showed a directional preference for extension while $72(48 \%)$ exhibited an inconclusive directional preference for either flexion or extension as described by McKenzie. With respect to the pattern of pain site distribution among the participants with PGP, the majority of the participants $(62.0 \%)$ had a bilateral presentation of pain. There was no significant difference in the mean age of the participants with respect to the McKenzie syndromes distribution among the participants with LBP and HBP.
Table 2. Distribution of the McKenzie syndromes and Pelvic girdle pain among the participants

Distribution of the McKenzie Syndromes among the participants with LBP $(N=230)$

\begin{tabular}{llll}
\hline & $\mathrm{n}(\%)$ & Mean Age \pm SD & p-level \\
\hline Derangement & $220(95.7 \%)$ & $27.0 \pm 5.9$ & \\
Dysfunction & NIL & & 1.000 \\
Postural & $10(4.3 \%)$ & $27.0 \pm 5.3$ & \\
\hline
\end{tabular}

Distribution of the McKenzie Syndromes among the participants with $\mathrm{HBP}(\mathbf{N}=\mathbf{8 6})$

\begin{tabular}{lll}
\hline & $\mathrm{n}(\%)$ & Mean Age \pm SD \\
\hline Derangement & $86(100 \%)$ & $26.2 \pm 5.2$ \\
Dysfunction & NIL & \\
Postural & NIL & \\
\hline
\end{tabular}

Distribution of pelvic girdle pain site among the participants with PGP $(\mathbf{N}=150)$

\begin{tabular}{|c|c|c|c|}
\hline & $\mathrm{n}(\%)$ & Mean Age \pm SD & p-level \\
\hline Unilateral & $57(38.0 \%)$ & $28.4 \pm 5.7$ & \\
\hline $\begin{array}{l}\text { Bilateral } \\
\alpha=0.05\end{array}$ & $93(62.0 \%)$ & $28.0 \pm 5.7$ & 0.6772 \\
\hline 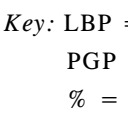 & $\begin{array}{l}\text { Back Pain; } \\
\text { ic Girdle Pain; } \\
\text { tage; }\end{array}$ & $\begin{array}{l}\mathrm{HBP}=\text { High Bac } \\
\mathrm{N} / \mathrm{n}=\text { Number } \\
\mathrm{SD}=\text { Standard de }\end{array}$ & $\begin{array}{l}\text { Pain; } \\
\text { iation }\end{array}$ \\
\hline
\end{tabular}

\section{DISCUSSION}

There is a significant difference in the characteristics of the participants involved in this study with respect to the demographic parameter of age. Participants with HBP had the lowest mean age while participants with PGP had the highest mean age. However, age seems not to be a determinant factor in the occurrence of the three McKenzie syndromes among the participants with LBP and HBP. With respect to age and occurrence of $\mathrm{BP}$ among pregnant women, there appear to be conflicts of opinion as to the relationship existing between the two factors of age and occurrence of BP. Some findings (Melzack and Belanger, 1989; Ostgaard et al, 1994; Heiberg and Aersath, 1997) indicated that maternal age is of no consequence to the problem of BP among pregnant women, while others (Bookhout and Boissonault, 1988; Breen et al, 1994) reported that back pain in pregnancy was associated with younger maternal age. However, others were of the opinion that pregnancy is an independent risk factor for the 
development of back pain in women (De Joseph and Cragin, 1998; Brynhildsen et al, 1998).

\section{Distribution of the McKenzie Syndromes, Posterior Pelvic Pain Site and Directional Preferences among the Participants}

Participants with derangement syndrome were in the overwhelming majority $(95.7 \%)$ among the participants with pregnancy-related LBP while all the participants with HBP had derangement syndrome only. This finding is contrary to the opinion of McKenzie (1980) who postulated that BP of postural origin was likely to be the most prevalent among pregnant women due to the postural adjustment that was frequent during pregnancy. However, this finding is consistent with that of Rath (1997) who reported that derangement syndrome and not postural syndrome was most prevalent among pregnant women based on clinical practice experience. The finding from the present study, showing a predominance of derangement syndrome among pregnant women, is similar to the findings among patients with LBP in the general population (McKenzie and May, 2003; Donelson, 2007; Ayanniyi et al, 2008). However, unlike in the general population a number of pregnant women in the present study with LBP showed a directional preference for flexion procedures which is indicative of the likely presence of anterior derangement, a sub-classification of derangement syndrome which has been attributed to hyperlordosis during pregnancy in women of childbearing age (McKenzie and May, 2003). The directional preferences for flexion and extension as exhibited by some of the participants with PGP may be an indication of possible co-existence of lumbar spine mechanical pathology with PGP in some of the participants (Ayanniyi et al, 2006, Vermani et al, 2010). This finding thus suggests that some of the participants with PGP may also benefit from the McKenzie protocol.

In the present study, only $4.3 \%$ of the pregnant women were classified as having LBP of postural origin while none was found among the participants with HBP. The dominance of derangement syndrome among pregnant women is supported by the finding of Weinreb et al (1989) that showed that bulges or herniation of lumbosacral discs is common in women of childbearing age which may give rise to derangement syndrome and not postural syndrome during pregnancy.

With respect to the distribution pattern of PGP site, participants with bilateral presentation were in the majority
$(61.7 \%)$ while $38.3 \%$ had a unilateral pain presentation. This finding is consistent with that of Albert et al (2000), where participants with bilateral pain presentation constituted the majority $(58.6 \%)$ and those with unilateral pain presentation constituted $41.4 \%$ of the study population. This is however at variance with the finding of Sturesson et al (1997), where pain site was nearly equally distributed with $34 \%$ of their participants having bilateral pain presentation compared to $37 \%$ with unilateral pain presentation, while $29 \%$ were inconclusive.

The mechanism leading to PGP is poorly understood, however, hormonal effects during pregnancy were implicated for promoting pelvic ligaments laxity which then gives rise to pelvic girdle relaxation in pregnancy (Kristiansson et al, 1999; Hansen et al, 1999). Studies by Petersen et al (1994) and Albert et al (1997), however, disputed any relationship between serum relaxin secretions and incidence of pelvic girdle pain in pregnancy. A systematic review conducted by Aldabe et al (2012), which assessed the level of evidence for the association between pregnancy-related PGP and relaxin levels during pregnancy, found the association to be low due to a number of confounding factors. However, according to Perkins et al (1998), most signs and symptoms of PGP are similar to that of sacroiliac joint dysfunction.

Limitation: The repeated movement testing component of the McKenzie method of assessment was modified to include fewer repetitions (two to three repetitions) per bout instead of the usual 10 repetitions per bout as employed in the general population with back pain. The overall effect of this modification on the efficiency or otherwise of the procedure is not clear.

The clinical implication of findings: The findings from this study suggest that the McKenzie method may have a useful role to play in the mechanical assessment, classification and management of pregnancy-related LBP, HBP, and PGP. The result of this study may guide decision making in the assessment and selection of interventions in pregnancy-related back pain and help improve physical therapy outcome.

\section{CONCLUSION AND RECOMMENDATIONS}

Derangement syndrome was the most prevalent among participants with pregnancy-related low back and high back pain while bilateral pelvic girdle pain presentation was the 
most common among the participants with pelvic girdle problem. Directional preferences were established for the McKenzie assessment procedure in the three back pain groups but more for participants with LBP and HBP . It is recommended that the McKenzie approach should be considered and used in the physical assessment of women with pregnancy-related back pain.

\section{Declaration of Interest}

The authors report no conflict of interest.

\section{Acknowledgement}

We specially thank all the pregnant women who participated in this study. We are grateful to the authorities of the various hospitals and the heads of department who gave us access to their clinical facilities.

\section{REFERENCES}

Albert H.B., Godskesen M., Westergaard J.G., Chard T., Gunn L. 1997. Circulating levels of relaxin are normal in pregnant women with pelvic pain. European Journal of Obstetrics, Gynecology, and Reproductive Biology 74(1): 19 - 22.

Albert H.B., Godskesen M., Westergaard J. 2000. Evaluation of Clinical tests used in classification procedures in pregnancyrelated pelvic joint pain. European Spine Journal 9: 161 - 166.

Aldabe D., Ribeiro D.C., Milosavljevic S., Bussey M.D. 2012. Pregnancy-related pelvic girdle pain and its relationship with relaxin levels during pregnancy: A systematic review. European Spine Journal 21(9): 1769-76. doi 10.1007 /s00586012-2162-x.

Ayanniyi O., Sanya A.O., Ogunlade S.O., Oni-Orisan M.O. 2006. Prevalence and pattern of back pain among pregnant women attending ante-natal clinics in selected health care facilities. African Journal of Biomedical Research 9: 149 - 156.

Ayanniyi O., Sanya A.O., Oni-Orisan M.O. 2008. Distribution of the three McKenzie syndromes among patients with low back pain in selected outpatient Physiotherapy facilities in Nigeria. African Journal of Medicine and Medical Sciences 37: 171-175.

Bookhout M.M., and Boissonault W.G. 1988. Physical therapy mangement of musculoskeletal disorders during pregnancy. In: E. Wilder (ed.) Obstetrics and Gynaecology Physical Therapy. New York: Churchill-Livingstone, pp. 17 - 62.

Breen T.W., Ransil B.J., Groves P.A., Oriol N.E. 1994. Factors associated with pain among postmenopausal women. Spine 23(7): $809-813$.

Brynhildsen J.O., Bjors E., Skarspgard C., Hammar M.L. 1998. Is hormone replacement therapy a risk factor for low back pain among postmenopausal women? Spine 23(7): 809 - 13.

Cyriax, J. 1978. Textbook of Orthopaedic Medicine. Volume 1:
Diagnosis of Soft Tissue Lesions. 7th Edition. London: Bailliera Tendal, pp.348 - 573.

DeJoseph J.F., Cragin L. 1998. Biomedical and feminist perspectives on low back pain during pregnancy. Nursing Clinic North America 33: 713-724.

Donelson R. 2007. Rapidly Reversible Low Back Pain. Hanover, New Hampshire. Self-Care First, LLC. 74 - 78.

Fritz J.M., Delitto A., Vignovic M., Busse R.G. 2000. Interrater reliability of judgments of the centralization phenomenon and status change during movement testing in patients with low back pain. Arch Physical Medical Rehabilitation 81(1): 57-61.

Hansen A., Jensen D.V., Wormslev M., Minck H., Johansen S., Larsen E.C. 1999. Symptom-giving pelvic girdle relaxation in pregnancy. II: Symptoms and clinical signs. Acta Obstetrician et Gynecologica Scandinavica 78: 111-115.

Heiberg E., Aersath S.P. 1997. Epidemiology of pelvic pain and low-back pain in pregnant woman. In: Vleeming A., Mooney V, Dorman T, Snijders C, Stoeckert R. (Eds.) Movement Stability and Low Back Pain. The essential role of the pelvis. New York: Churchill Livingstone.

Hipp R., Gradinger R., Flock K., Opitz G., Hipp E. 1989. Backache causes, diagnosis and therapy. Fortschritte der Medizin 107(18): 36; 39-40; 43- 44.

Kristiansson P., Svardsudd K., Von Schoultz B. 1999. Reproductive hormones and aminoterminal propeptide of type III procollagen in serum as early markers of pelvic pain during late pregnancy. American Journal of Obstetrics and Gynecology 180: 128 - 34.

Lile J., Perkins J., Hammer R.L., Loubert P.V. 2003. Diagnostic and management strategies for pregnant women with back pain. JAAPA 16: 31-44.

MacFayden I.F. 1989. Maternal aspects of pregnancy. In: Turnbull A. and Chamberlain G. (eds.) Obstetrics. Edinburg: Churchill Livingstone, pp.151 - 172.

McKenzie R.A. 1980. Treat Your Own Back. Waikanae, New Zealand: Spinal Publication, pp.67 - 69.

McKenzie R.A. 1990. The Cervical and Thoracic Spine Mechanical Diagnosis and Therapy. Waikanae, New Zealand: Spinal Publication, pp. xix-xxiii; 1-56.

McKenzie R.A. and May S. 2003. The Lumbar Spine Mechanical Diagnosis and Therapy $2^{\text {nd }}$ edition. Waikanae, New Zealand: Spinal Publication, pp.139 - 148.

Melzack R., Belanger E. 1989. Labour pain: correlation with menstrual pain before and during pregnancy. Pain 36(2): 2259 .

Mogren I.M., Pohjanen A.I. 2005. Low back pain and pelvic pain during pregnancy: prevalence and risk factors. Spine 30(8): 983-91.

Ostgaard H.C., Roos-Hansson E., Zetherstrom G. 1996. Regression of back and posterior pelvic pain after pregnancy. Spine 21(23): 2777 - 80.

Ostgaard H.C., Zetherstrom G., Roos Hansson E., Svanberg B. 1994. Reduction of back and posterior pelvic pain in pregnancy. Spine 19: 894 - 900. 
Perkins J., Hammer R.L., Loubert P.V. 1998. Identification and management of pregnancy-related low back pain. Journal of Nurse-Midwifery 43(5): 331 - 40.

Petersen, L.K., Hvidman L., Uldbjerg N. 1994. Normal serum relaxin in women with disabling pelvic pain during pregnancy. Gynecology and Obstetrics Investigations 38: 21 - 23.

Rath, J.D. 1997. Mechanical low back pain in pregnancy: Preliminary findings in a consecutive case series investigation. Abstract of proceedings. 5th McKenzie Institute International Conference, Philadelphia Pennsylvania, 12 - 14 September 1997. Journal of McKenzie Institute USA 5(3): 6-30.

Razmjou H., Kramer J.F., Yamada R. 2000. Intertester reliability of the McKenzie evaluation in assessing patients with mechanical low back pain. Journal of Orthopaedic Sports Physical Therapy 30(7): 368-83.

Sturesson B., Uden G., Uden A. 1997. Pain pattern in pregnancy and "catching" of leg in pregnant women with posterior pelvic pain. Spine 22(16): 1880 - 1884.

Vermani E., Mittal R., Weeks A. 2010. Pelvic girdle pain and low back pain in pregnancy: a review. Pain Practice 10(1): 60-71.

Vleeming A., Albert H.B., Ostgaard H.C., Sturesson B., Stuge B. 2008. European guidelines for the diagnosis and treatment of pelvic girdle pain. European Spine Journal 17: 794-819.

Weinreb J.C., Wolbarsht L.B., Cohen J.M., Brown C.E. and Maravilla K.R. 1989. Prevalence of lumbosacral intervertebral disk abnormalities on MR Images in pregnant and asymptomatic non-pregnant women. Radiology 170: 125-128.

Wu W.H., Meijer O.G., Uegaki K., Mens J.M.A., van Dieen J.H., Wuisman P.I.J.M., Ostgaard H.C. 2004. Pregnancyrelated pelvic girdle pain, (PPP) terminology, clinical presentation, and prevalence. European Spine Journal 13: 575-589. 